\title{
Pediatric health care professionals' vaccine knowledge, awareness and attitude: a survey within the Italian Society of Pediatric Allergy and Immunology
}

Elisabetta Del Duca ${ }^{1 *}$ (D), Loredana Chini ${ }^{1}$, Simona Graziani ${ }^{1}$, Mayla Sgrulletti ${ }^{1,2}$, Viviana Moschese ${ }^{1,3}$ and with the Italian Pediatric Immunology and Allergology Society (SIAIP) Vaccine Committee

\begin{abstract}
Background: Physicians play a key role in driving vaccine acceptance and their recommendations are crucial to address vaccine hesitancy. The aim of the study was to assess knowledge, awareness and attitude of Italian Pediatric Health Care Professionals (pHCPs) on vaccinations.

Methods: An anonymous on-line questionnaire was developed within the Vaccine Committee of Italian Society of Pediatric Allergy and Immunology (SIAIP) and spontaneously completed by 231 Pediatricians and Pediatric Nurses (PN).

Results: An accurate vaccine education was reported by $70 \%$ of pediatricians and $13 \%$ of PN but $11 \%$ of pediatricians versus $26 \%$ of PN consult social media instead of scientific sources for their vaccine update. The investigation on the pHCPs attitudes to vaccination in a personal and family setting highlights poor adherence to vaccinations. Only $63 \%$ of pediatricians versus $16 \%$ of PN $(p<0.0001)$ annually received the Flu vaccine. In their family setting $93 \%$ of pediatricians versus $51 \%$ of PN recommended all vaccinations ( $p<0.0001$ ). Anti-flu, anti-rotavirus, anti-zoster and anti-pneumococcal vaccines were not regularly recommended by all pHCPs due to doubts of uselessness (55\% of pediatricians versus $40 \%$ of PN) and preference for "natural immunity" (44\% of pediatricians versus $40 \%$ of PN).
\end{abstract}

Conclusions: Our results indicate that pHCPs' attitude and confidence in regards to vaccines remain suboptimal. Current COVID-19 pandemic and the rapid development of vaccines could increase vaccine hesitancy. Due to the documented pHCPs' influence in the parental decision, educational interventions are needed to improve their level of knowledge and counselling skills in order to address parental vaccine hesitancy and to maintain continuity of immunization services.

Keywords: Vaccine hesitancy, Vaccine knowledge, Vaccine attitudes, Pediatric health care professionals, Channel sources

\section{Background}

The World Health Organization (WHO) recommends vaccine coverage threshold of $95 \%$ to prevent and control the circulation of viruses and bacteria in the community and to reach the so-called "herd immunity". In

\footnotetext{
* Correspondence: elisabetta.delduca@tin.it

'Pediatric Immunopathology and Allergology Unit, Policlinico Tor Vergata, University of Rome Tor Vergata, Viale Oxford 81, 00133, Rome, Italy Full list of author information is available at the end of the article
}

order to contribute to the implementation of immunization programs in all countries an European Immunization Plan (European Region Vaccine Action Plan) has been developed [1]. In Italy, since 2005, the Ministry of Health has published the National Immunization Prevention Plan (PNPV) to support a uniform vaccination strategy across all regions; nevertheless, in the last decade, vaccination coverage showed a decline in almost every region of the country, suggesting low

(c) The Author(s). 2021 Open Access This article is licensed under a Creative Commons Attribution 4.0 International License, which permits use, sharing, adaptation, distribution and reproduction in any medium or format, as long as you give appropriate credit to the original author(s) and the source, provide a link to the Creative Commons licence, and indicate if changes were made. The images or other third party material in this article are included in the article's Creative Commons licence, unless indicated otherwise in a credit line to the material. If material is not included in the article's Creative Commons licence and your intended use is not permitted by statutory regulation or exceeds the permitted use, you will need to obtain permission directly from the copyright holder. To view a copy of this licence, visit http://creativecommons.org/licenses/by/4.0/ The Creative Commons Public Domain Dedication waiver (http://creativecommons.org/publicdomain/zero/1.0/) applies to the data made available in this article, unless otherwise stated in a credit line to the data. 
vaccine awareness and increased parental vaccine hesitancy.

For example, polio vaccine coverage rate fell from $96.1 \%$ in 2011 to $93.3 \%$ in 2016 at 24 months of age [2] and between 2013 and 2015 the vaccine coverage for measles, mumps and rubella (MMR) fell from 90 to $85 \%$, at 24 months of age [2]. Furthermore, between January and December 2017, a severe measles outbreak occurred in Italy, with 4885 measles cases, including 4 deaths [3].

To increase vaccination rates, a novel National Vaccine Prevention Plan (PNPV 2017-2019) [4] extended the list of recommended vaccines and introduced new target populations. Vaccines against Neisseria meningitidis $B$, rotavirus and varicella were recommended for children; tetravalent meningococcal vaccine (ACWY135) and a booster dose of anti-polio for adolescents, as well as human papillomavirus vaccine (HPV) for male adolescents; last but not least conjugated (PCV) or polysaccharide pneumococcal vaccines (PPV) and zoster vaccination for subjects over 65 years old and atrisk categories. Moreover, such vaccines were included into the Essential Care Levels of Assistance (LEA) and information campaigns were promoted. To achieve the goals of the PNPV, an urgent Decree-Law (No.73 June 2017), subsequently converted into a law (No.119 July 2017) approved 10 mandatory vaccines, such as pertussis, measles, mumps, rubella, varicella, Haemophilus influenzae type $b$, diphtheria, tetanus, hepatitis $\mathrm{B}$ and polio vaccines [5]. According to the law, these vaccines are mandatory for school admission. In 2018, a slight vaccine coverage increase was observed in some Italian regions, but results were far from the WHO recommended threshold: among children at 24 month of age, vaccination coverage for diphtheria, polio, tetanus barely reached 95\% whereas for hepatitis B it was still below $95 \%$. Polio vaccine uptake exceeded $95 \%$ in 14 regions, whereas in 7 regions was less than 93\% [2]. As reported in a recent Italian survey, parental vaccine hesitancy due to safety and efficacy is still high [6].
Several studies have underlined that physicians' recommendation is a key determinant in the parental decision-making process to vaccinate their children [7], also a favorable parent opinion is strongly influenced by a favorable physician opinion $[8,9]$. The aim of our study was to assess vaccine knowledge and awareness among Italian Pediatric Health Care Professionals (pHCPs) such as Pediatricians and Pediatric Nurses $(\mathrm{PN})$, as well as their personal and family vaccination adherence, to draw a real picture of their attitude.

\section{Methods}

The Vaccine Committee of the Italian Society of Pediatric Allergy and Immunology (SIAIP) has implemented an anonymous, online and multiple choice questionnaire for pediatricians and $\mathrm{PN}$ published on the official website of the Society (www.siaip.it). The questionnaire included 31 items consisting of three major sections and was delivered by Google Supplementary Modules. The first section contained questions to define respondents' training in vaccinology and their channel sources for vaccine-related updates. The second section aimed to assess their perception on parental vaccine concerns and hesitancy, their attitude to discussing the vaccine issue with families as well as their awareness regarding vaccine uptake in childhood. The last section analyzed their personal and family vaccine attitudes.

One hundred and thirty seven pediatricians, belonging to the SIAIP and to the Italian Health System, and 94 PN spontaneously answered to the online survey.

Statistical analysis was performed using Fisher's exact test and Chi-Square test with a $p$ value $\leq 0.05$ considered significant.

\section{Results}

pHCPs' training in vaccinology and channel sources for vaccine update

The answers to the first section showed that $96 / 137$ (70\%) pediatricians compared to $12 / 94$ (13\%) PN

Table 1 Main determinants of parental vaccine hesitancy referred by 198/231 Pediatric Health Care Professionals (pHCPs)

\begin{tabular}{lll}
\hline & Total 198 & \% \\
\hline Concern about vaccine adverse reactions & 145 & $73 \%$ \\
Concern about development of autism spectrum disorders & 90 & $46 \%$ \\
Concern about development of autoimmune diseases & 75 & $43 \%$ \\
Doubts on usefulness and effectiveness of vaccines & 38 & $38 \%$ \\
Concern about adjuvants adverse effects & 36 & $19 \%$ \\
Concern about pain and stress induced by vaccination & 32 & $18 \%$ \\
Preference for the development of natural immunity & 29 & $16 \%$ \\
Concern about the risk to get the disease by the vaccination & 29 & $15 \%$ \\
Lack of trust in pharmaceutical companies & 23 & $15 \%$ \\
Too expensive & $12 \%$ \\
\hline
\end{tabular}




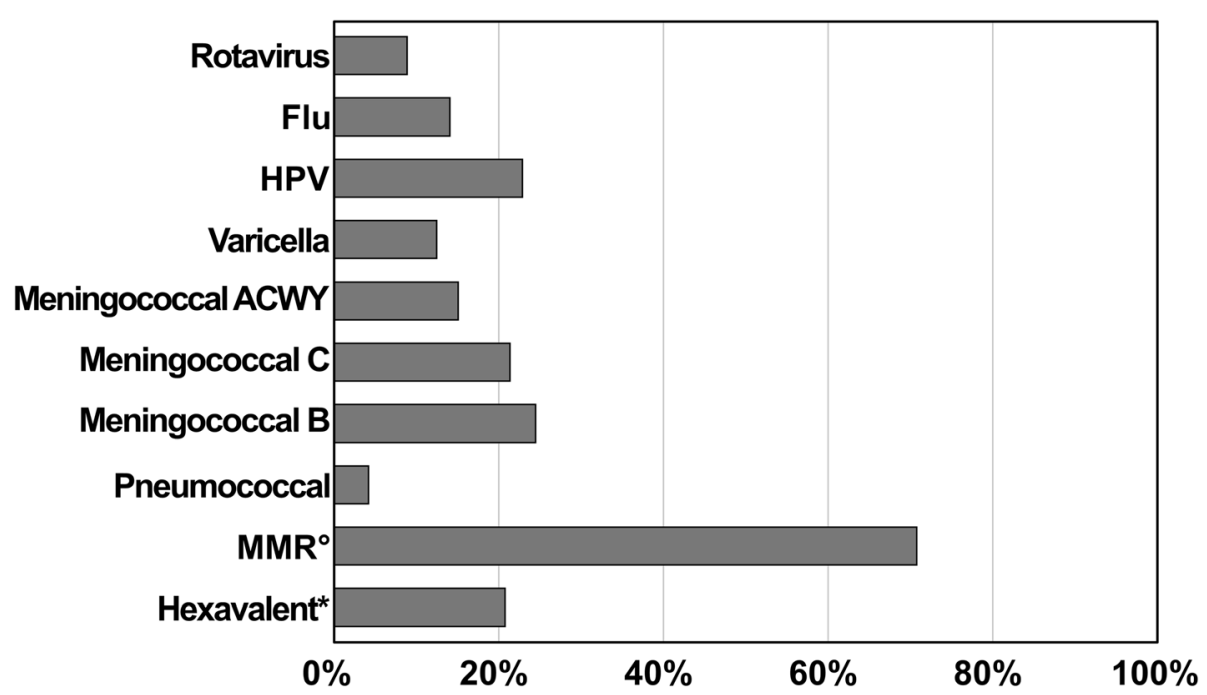

Fig. 1 Parental concern and refusal to specific vaccines as reported by Pediatricians and Pediatric nurses ( ${ }^{\circ}$ Measles-Mumps-Rubella; ${ }^{*}$ PolioDifteria-Tetanus-Pertussis-Hepatitis B-Haemophilus influenzae type b)

considered their training appropriate $(p<0.0001)$. 109/137 (80\%) pediatricians and 66/94 (70\%) PN used the Italian Health Minister and the National Institute of Health (ISS) websites as well as scientific literature for their update. Conversely, 15/137 (11\%) pediatricians and 24/94 (26\%) PN consulted channel sources such as social networks, blogs or media.
pHCPs' perception of parents' vaccine concern and hesitancy and the dialogue with families

One hundred ninety eight out of 231 (86\%) pHCPs declared to perceive an increase in parental concern on vaccine safety/efficacy, with no significant differences between pediatricians and PN (data not shown).

Main concerns and related vaccines are shown in Table 1 and in Fig. 1, respectively.

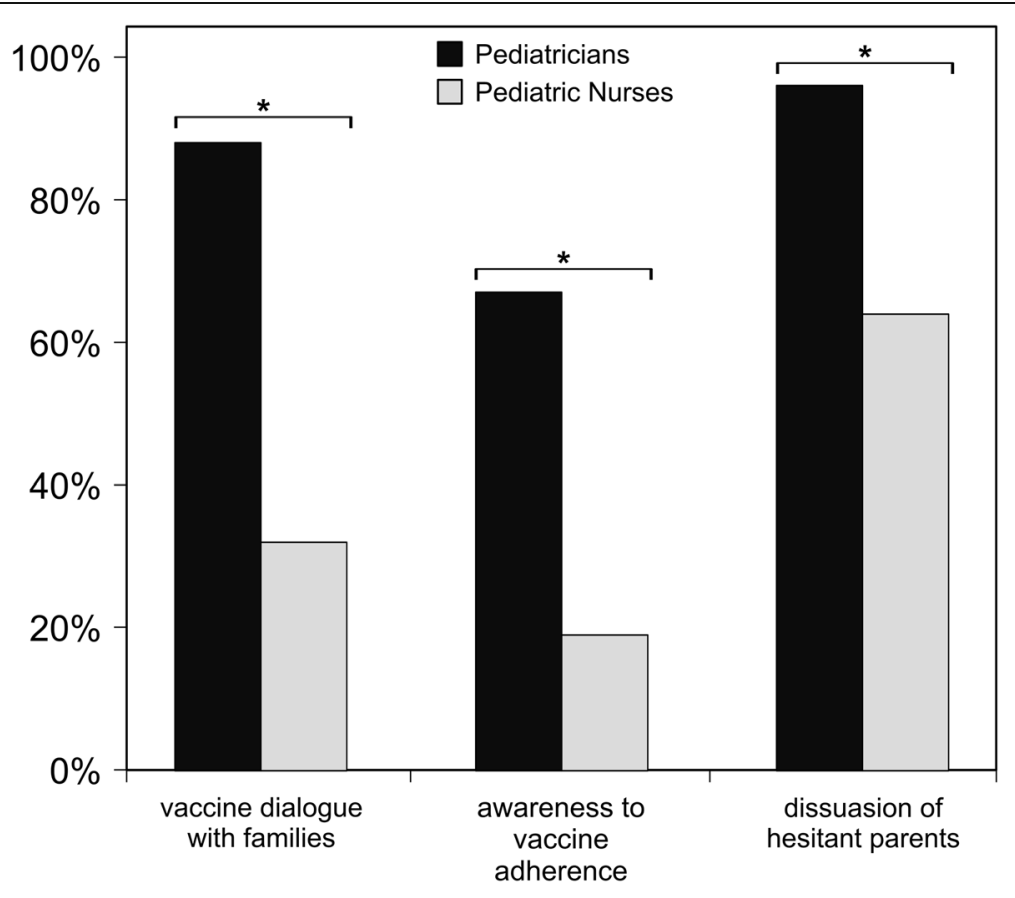

${ }^{*} p<0.0001$

Fig. 2 The behaviours of Pediatricians and Pediatric Nurses on parental vaccination making process with regard to vaccine dialogue with families, awareness to vaccine adherence and dissuasion of hesitant parents 


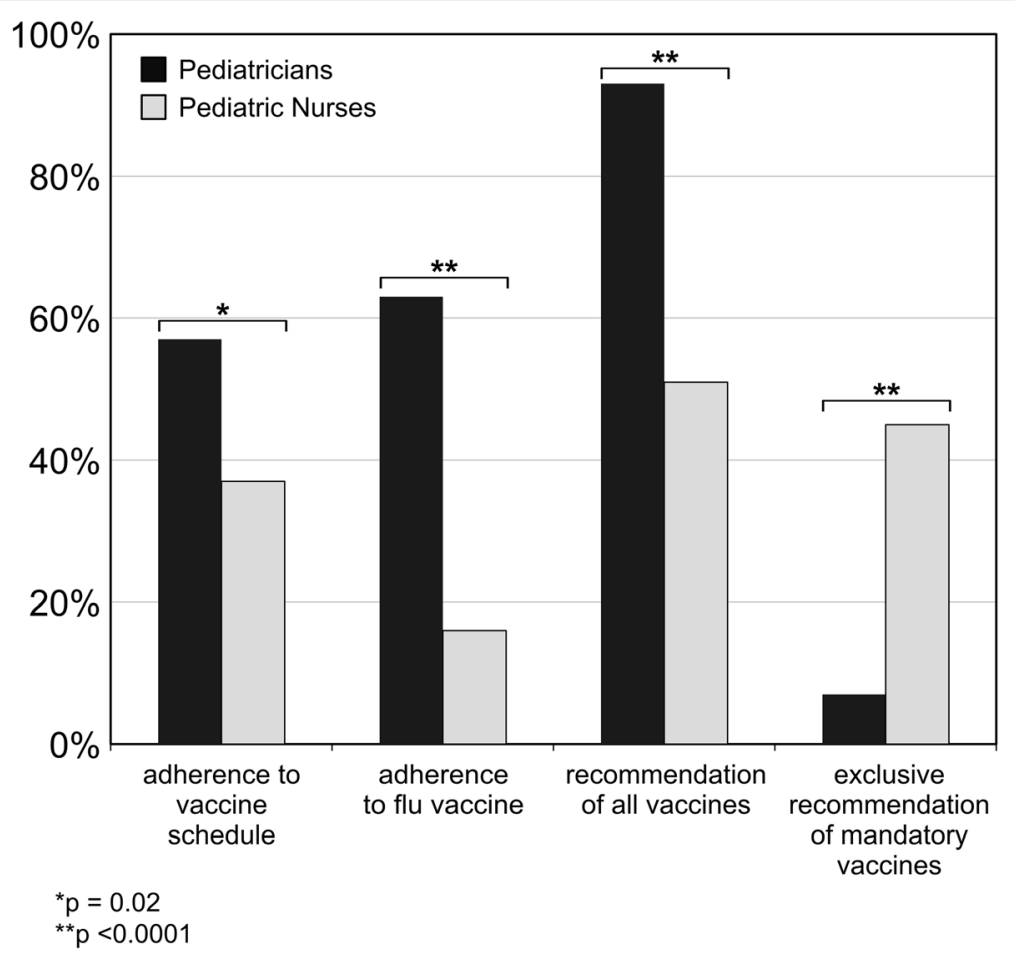

Fig. 3 Personal and family vaccine attitude of Pediatricians and Pediatric Nurses for adherence to routine vaccine schedule, adherence to flu vaccine, recommendation of all vaccines and exclusive recommendation of mandatory vaccines in the family setting

A continuous vaccine communication with the families was reported by $121 / 137(88 \%)$ pediatricians versus 30/94 (32\%) PN ( $p<0.0001)$ (Fig. 2).

Moreover, 92/137 (67\%) pediatricians claimed to regularly verify children's adherence to vaccine schedules versus 18/94 (19\%) PN ( $p<0.0001)$; also, 132/137 (96\%) pediatricians versus 60/94 (64\%) PN $(p<0.0001)$ tried to dissuade the hesitant parents (Fig. 2).

\section{Vaccine attitude of pediatric health care professionals in personal and family setting}

Adherence to vaccination schedule has been reported to be constant in 78/137 (57\%) pediatricians versus 35/94 (37\%) PN ( $p=0.02)$ (Fig. 3). On the question "Do you yearly get flu vaccine?” 86/137 (63\%) pediatricians versus 15/94 (16\%) PN replied "yes" ( $p<0.0001)$ (Fig. 3).

In the family setting, $127 / 137$ (93\%) pediatricians and 48/94 (51\%) PN promoted vaccine recommendations $(p<0.0001)$, with 10/137 (7\%) pediatricians and 42/94 (45\%) PN recommending only the mandatory vaccines $(p<0.0001)$ (Fig. 3).

Anti-flu, anti-rotavirus, anti-zoster and antipneumococcal vaccines were not regularly recommended. The main reasons for vaccine refusal were doubts of uselessness in 75/137 (55\%) pediatricians versus 38/94 (40\%) PN and preference for "natural" immunity in 60/137 (44\%) pediatricians versus 38/94 (40\%) PN. Moreover 4/94 (4\%) PN declared No-vax position.

\section{Discussion}

Vaccine hesitancy is an important issue that needs to be addressed to achieve effective control over vaccinepreventable diseases [10]. Parental concerns are mainly related to the lack of confidence on vaccine efficacy and safety, as well as to misinformation (fake news) regarding adverse effects. The latter includes, in particular, the presumed and disavowed link between vaccines and autism, allergies or autoimmune diseases $[6,11]$ and is derived from the growing opposition movements due to ideological reasons (no-vax) [11].

Among the possible reasons for vaccine hesitancy the lack of appropriate recommendations with simple and clear information by pHCPs could also play a role [12]. Therefore, univocal information and a strong familyHCP relationship based on awareness and dialogue are essential to address vaccine concerns and improve vaccine uptake $[7,8]$.

It is a fact that after the Decree-law of June and the law of July 2017, vaccine coverage against tetanus, polio, MMR, pneumococcal and meningococcal $\mathrm{C}$, showed respectively a $0.9,1.2,4.4,2.4$ and $2.4 \%$ increase in the birth cohort of 2017 compared to 2016 [13]. 
Nevertheless, according to data reported by National Institute of Health, in Italy, between January 2018 and December 2018, measles cases were 2526, including 8 deaths [3], while those reported in 2019 were 1627 with more than half of them in Lombardy and Lazio regions. The average age of the affected patients was 30 years but the highest incidence was reported in children $<5$ years of age. Indeed, among 174 cases including 64 children < 1 year (136.9 cases/1.000.000), $86 \%$ of patients were not vaccinated [3]. Of note, 96/1627 cases have been reported among health care providers. Between January and August 2020, measles cases were 101 in 12 regions with an average age of 33 years. Again, in 2020 the highest incidence has been reported in children $<5$ years of age (4.6 cases/1000000) and five cases reported in children $<1$ year of age (11.4 cases/1000000). $93 \%$ of all patients were not vaccinated with 12 cases among health care professionals. Although these data show a higher trend in vaccine coverage, in addition to legislative measures, other strategies are necessary to improve vaccine uptake [14]. From a cross-sectional survey aimed to estimate vaccine hesitancy and its determinants on 3130 parents of children aged 16-36 months, emerged the fact that, in Italy, safety concerns are the main reported reasons for refusing or interrupting vaccinations [6]. Accordingly, the majority of pHCPs respondents reported that doubts on safety and efficacy of vaccinations were the main reasons for parental refusal and hesitation. Inadequate and mostly negative information by media and websites have certainly increased parental vaccinehesitancy. An analysis conducted on 153 YouTube videos on vaccination in children indicated that, in $50 \%$ of the cases, the information on immunization were not pro-vaccine and rather discordant with scientific literature; another observational study reported that YouTube videos that disapproved vaccination were the most shared by the viewers $[15,16]$. The European Center for Disease Prevention and Control (ECDC) and other public health organizations $[17,18]$ published guidelines for health care professionals to provide them with tools to

Table 2 Main implementation strategies to improve Pediatric Health Care Professionals (pHCPs) knowledge and awareness

Provide appropriate vaccine knowledge and training on vaccinology during academic years

Favor easy and free access for vaccine update

Plan continuing educational interventions

Ensure tools to empower communication skills and basic techniques of relationship

Organize intersociety meetings to update of vaccine guidelines

Qualify Vaccine Centers with appropriate structural and administrative supports

Improve use of digital technology for vaccine programs optimize the effectiveness of vaccine discussion with parents. In this regard, our data show that PN, compared to pediatricians, are less conscious to track children's vaccine schedule and provide parents with factual information about vaccine programs. However, vaccine education and training was mostly considered inappropriate by pediatricians and PN during their academic and professional years. The observation that approximately $10 \%$ of pediatricians and $20 \%$ of PN consult social media rather than scientific sources for vaccine update, urges the need for improving educational and counselling skills among pHCPs. Studies from other WHO regions recommend vaccine education programs to increase health professional's knowledge and awareness to enhance vaccination coverage [19-22].

In our study we found poor adherence of pediatricians and $\mathrm{PN}$ to vaccine schedule, since only $57 \%$ of pediatricians and $37 \%$ of PN respond to vaccine recommendations. In particular, only $63 \%$ of pediatricians and $16 \%$ of $\mathrm{PN}$ receive the annual flu vaccine. Things do not go better for $\mathrm{PN}$ in the family setting, since a significant proportion compared to pediatricians ( $45 \%$ versus $7 \%$; $p<$ $0,0001)$, recommend only selected vaccines due to their concerns on vaccine efficacy and their preference for the development of "natural" immunity. It goes without saying, this behaviour might affect parental decision- making process to vaccinate their children.

Although there are significant differences between pediatricians and $\mathrm{PN}$, our current findings show that vaccine attitude and knowledge of pediatric health care professionals remains suboptimal mainly due to misinformation and concerns regarding vaccine safety and efficacy.

In Table 2 we indicate main implementation strategies to improve vaccination management among pHCPs. Educational themes represent a priority, but also communication skills and participatory practices should be encouraged. In fact counselling skills are key elements to tackle vaccine hesitancy as recently recommended by the National Institute of Health [23]. Last but not least, an appropriate environment with structural, administrative and digital supports would motivate health professionals and positively affect their efforts to sustain vaccine uptake.

We acknowledge that the assessment of pHCPs' knowledge, awareness and attitude on a limited sample size should be complemented by further data collection to allow a more comprehensive generalizability of our findings. However, since the questionnaire was filled in by voluntary and anonymous pediatricians and PN that have a direct counseling role we believe that this survey may represent a useful evidence base of pHCPs' vaccine confidence. Indeed, healthcare workers provide role modeling for preventive behaviors and the bulk of 
immunization strategies. Covid-19 pandemic makes such educational interventions even more urgent due to the occurrence of disruption or delay in routine vaccination programs with the risk of re-occurrence of vaccine preventable disease. Further, with the introduction of Covid-19 vaccine a clear and detailed information to healthcare workers is to be prioritized since a high rate of vaccine skepticism has been observed, including health care workers [24]. In this regard the Ministry of Health has issued a document with 2020-2021 vaccine recommendations. Flu vaccination has been offered actively and free of charge to all children from 6 months to 6 years of age and recommended in all pediatricians and pregnant women.

To strengthen vaccine uptake distinct international and national organizations as well as scientific societies including the Italian Society of Pediatric Allergy and Immunology (SIAIP), can give a significant contribution to patrol clear and simple information to all stakeholders. In this regard in October 2019 the European Academy of Allergy and Clinical Immunology (EAACI) has produced a video to spread vaccination culture worldwide [25]. Furthermore, in March 2020, the WHO has published a document with guiding principles to maintain continuity of immunization services [26] and, in June 2020, the SIAIP provided a Consensus Statement to offer a rationale to help guide decision-making in the management of children and adolescents with allergic or immunologic diseases [27].

\section{Conclusions}

Although the sample size was limited, our data show the urgent need to design and plan educational interventions to improve Health Care Professionals' vaccine knowledge and to ensure optimal acquisition of communication skills for vaccine uptake. This is particularly true at Covid-19 pandemic time where key protection of children and the entire community against serious vaccinepreventable disease can be governed by inclusive vaccine compliance.

\footnotetext{
Abbreviations

ECDC: European Center for Disease Prevention and Control; LEA: Essential Care Levels of Assistance; MMR: Measles, Mumps and Rubella; PCV: Pneumococcal conjugate vaccine 13; pHCPs: Pediatric Health Care Professionals; PN: Pediatric Nurses; PNPV: National Immunization Prevention Plan; PPV: Pneumococcal polysaccharide vaccine 23; SIAIP: Italian Society of Pediatric Allergy and Immunology; WHO: World Health Organization
}

\section{Acknowledgments}

The Vaccine Committee of the Italian Society of Pediatric Allergy and Immunology thanks all SIAIP members for their contribution to the survey.

\section{Authors' contributions}

VM and LC designed the study, read and approved the manuscript. EDD is the corresponding author, performed data analysis and wrote the draft. SG and MS performed data analysis and contributed to writing. SIAIP Vaccine
Committee participated to the study design and approved the manuscript. The author(s) read and approved the final manuscript.

\section{Funding}

This research did not receive any specific grant from funding agencies in the public, commercial, or not-for-profit sectors.

\section{Availability of data and materials}

Data are available from the corresponding author on reasonable request.

\section{Declarations}

Ethics approval and consent to participate

The study was approved by the Ethics Committee of the Italian Society of Pediatric Allergy and Immunology (SIAIP) and consent for publication was not required since the study was anonymous.

Consent for publication

Consent for publication was not required since the study was anonymous.

\section{Competing interests}

None declared.

\section{Author details}

${ }^{1}$ Pediatric Immunopathology and Allergology Unit, Policlinico Tor Vergata, University of Rome Tor Vergata, Viale Oxford 81, 00133, Rome, Italy. ${ }^{2} \mathrm{PhD}$ program in Immunology, Molecular Medicine and Applied Biotechnology, University of Rome Tor Vergata, Rome, Italy. ${ }^{3}$ Department Saint Camillus International University of Health and Medical Sciences, Rome, Italy. ${ }^{4}$ Department of Pediatrics, Fondazione IRCCS Ca' Granda, Ospedale Maggiore Policlinico, Milan, Italy. ${ }^{5}$ UOC of Pediatrics and Neonatology, "Monsignor A.R. Dimiccoli" Hospital, Barletta, Italy. ${ }^{6}$ Unit of Pediatrics, "Policlinico-Giovanni XXIII" Hospital, University of Bari, Bari, Italy. 'Department of Public Health and Pediatrics, Regina Margherita Children Hospital, University of Turin, Turin, Italy. ${ }^{8}$ Pediatric Haematology, Milano-Bicocca University, Monza, Italy. ${ }^{9}$ Innovation and Clinical Pathways Unit, Bambino Gesù Children's Hospital, IRCCS, Rome, Italy. ${ }^{10}$ Division of Pediatric Immunology and Rheumatology, Department of Pediatrics, Sapienza University of Rome, Rome, Italy.

${ }^{11}$ Pediatric Clinic, Fondazione IRCCS Policlinico San Matteo, University of Pavia, Pavia, Italy.

Received: 30 December 2020 Accepted: 29 May 2021

Published online: 09 September 2021

References

1. The World Health Organization. Regional Office for Europe. European Region Vaccine Action Plan 2015-2020. http://www.euro.who.int.

2. National Institute of Health. https://www.epicentro.iss.it/vaccini/dati_Ita

3. National Institute of Health, Measles Weekly Report. http://www.epicentro. iss.it/morbillo/infografica.

4. Signorelli C, Guerra R, Siliquini R, Ricciardi W. Italy's response to vaccine hesitancy: an innovative and cost effective National Immunization Plan based on scientific evidence. Vaccine. 2017;35(33):4057-9. https://doi.org/1 0.1016/j.vaccine.2017.06.011.

5. Ministero della Salute. Decreto legge 7 giugno 2017, n. 73. Disposizioni urgenti in materia di prevenzione vaccinale, come modificato dalla Legge di conversione 31 luglio 2017, n. 119. https://www.trovanorme.salute.gov.it/ norme/dettaglioAtto?id=60201.

6. Giambi C, Fabiani M, D'Ancona F, Ferrara L, Fiacchini D, Gallo T, et al. Parental vaccine hesitancy in Italy - Results from a national survey. Vaccine. 2018;36(6):779-87

7. Boes L, Boedeker B, Schmich P, Wetzstein M, Wichmann O, Remschmidt C. Factors associated with parental acceptance of seasonal influenza vaccination for their children - a telephone survey in the adult population in Germany. Vaccine. 2017;35(30):3789-96. https://doi.org/10.1016/j.vaccine.2 017.05.015.

8. Kennedy A, Basket M, Sheedy K. Vaccine attitudes, concerns, and information sources reported by parents of young children: results from the 2009 HealthStyles survey. Pediatrics. 2011;127(suppl 1):S92-9. https://doi. org/10.1542/peds.2010-1722N. 
9. Facciolà A, Visalli G, Orlando A, Bertuccio MP, Spataro P, Squeri R, et al. Vaccine hesitancy: an overview on parents' opinions about vaccination and possible reasons of vaccine refusal. J Public Health Res. 2019;8(1):1436. https://doi.org/10.4081/jphr.2019.1436.

10. Crenna S, Osculati A, Visonà SD. Vaccination policy in Italy: an update. J Public Health Res. 2018;7(3):1523. https://doi.org/10.4081/jphr.2018.1523.

11. Costa-Pinto J, Willaby HW, Leask J, Wood N, Marshall H, Danchin M. Vaccine discussions with parents: the experience of Australian paediatricians. J Paediatr Child Health. 2017;53(9):855-61. https://doi.org/10.1111/jpc.13587.

12. Edwards KM, Hackell JM. Committee on infectious diseases, the committee on practice and ambulatory medicine. Countering vaccine hesitancy. Pediatrics. 2016;138(3):e20162146.

13. D'Ancona F, D'Amario C, Maraglino F, Rezza G, Ricciardi W, lannazzo S. Introduction of new and reinforcement of existing compulsory vaccinations in Italy: first evaluation of the impact on vaccination coverage in 2017. Euro Surveill. 2018;23(22):1800238.

14. Siciliani L, Wild C, McKee M, Kringos D, Barry MM, Barros PP, et al. Strengthening vaccination programmes and health systems in the European Union: A framework for action. Health Policy. 2020;124(5):511-8. https://doi.org/10.1016/j.healthpol.2020.02.015.

15. Keelan J, Pavri-Garcia V, Tomlinson G, Wilson K. YouTube as a source of information on immunization: a content analysis. JAMA. 2007:298(21):24824. https://doi.org/10.1001/jama.298.21.2482.

16. Covolo L, Ceretti E, Passeri C, Boletti M, Gelatti U. What arguments on vaccinations run through YouTube videos in Italy? A content analysis. Hum Vaccin Immunother. 2017;13(7):1693-9. https://doi.org/10.1080/21645515.2 017.1306159 .

17. European Centre for Disease Prevention and Control. Let's talk about protection. En-hancing childhood vaccination uptake. Communication guide for healthcare providers. Stockholm: European Centre for Disease Prevention and Control; 2016. https:/ecdc.europa.eu/sites/portal/files/ media/en/publications/Publications/lets-talk-about-protection-vaccinationguide.pdf. Accessed 30 Dec 2017

18. Shen SC, Dubey V. Addressing vaccine hesitancy, clinical guidance for primary care physicians working with parents. Can Fam Physician. 2019; 65(3):175-81.

19. Peterson P, Meurice F, Stanberry LR, Glismann S, Rosenthal SL, Larson HJ. Vaccine hesitancy and healthcare providers. Vaccine. 2016;34(52):6700-6. https://doi.org/10.1016/j.vaccine.2016.10.042.

20. Chotta NAS, Mgongo M, Uriyo JG, Msuya SE, Stray-Pedersen B, StrayPedersen A. Awareness and factors associated with health care Worker's knowledge on rubella infection: a study after the introduction of rubella vaccine in Tanzania. Int J Environ Res Public Health. 2019;16(10):1676. https://doi.org/10.3390/ijerph16101676.

21. Yuan $Q$, Wang F, Zheng H, Zhang G, Miao N, Sun X, et al. Hepatitis B vaccination coverage among health care workers in China. PLoS One. 2019; 14(5):e0216598.

22. Chellapandian P, Myneni S, Ravikumar D, Padmanaban P, James KM Kunasekaran VM. Knowledge on cervical cancer and perceived barriers to the uptake of HPV vaccination among health professionals. BMC Womens Health. 2021;21 (1):65. https://doi.org/10.1186/s12905-021-01205-8.

23. Possenti V, Luzi AM, Colucci A, De Mei B. Communication and basic health counselling skills to tackle vaccine hesitancy. Ann Ist Super Sanità. 2019; 55(2):195-9. https://doi.org/10.4415/ANN_19_02_12.

24. Dror AA, Eisenbach N, Taiber S, Morozov NG, Mizrachi M, Zigron A, et al. Vaccine hesitancy: the next challenge in the fight against COVID-19. Eur J Epidemiol. 2020;35(8):775-9. https://doi.org/10.1007/s10654-020-00671-y.

25. "EAACl explains vaccination" https://www.youtube.com/watch?v= mRyDSalnaBY

26. COVID-19: Strategic planning and operational guidance for maintaining essential health services during an outbreak. 20 March 2020. http://www. who.int/emergencies/diseases/novel-coronavirus-2019/technical-guidance

27. Cardinale F, Ciprandi G, Barberi S, Bernardini R, Caffarelli C, Calvani M, et al. Consensus statement of the Italian society of pediatric allergy and immunology for the pragmatic management of children and adolescents with allergic or immunological diseases during the COVID19 pandemic. Ital J Pediatr. 2020;46(1):84. https://doi.org/10.1186/s13052-020-00843-2.

\section{Publisher's Note}

Springer Nature remains neutral with regard to jurisdictional claims in published maps and institutional affiliations.

\section{Ready to submit your research? Choose BMC and benefit from:}

- fast, convenient online submission

- thorough peer review by experienced researchers in your field

- rapid publication on acceptance

- support for research data, including large and complex data types

- gold Open Access which fosters wider collaboration and increased citations

- maximum visibility for your research: over $100 \mathrm{M}$ website views per year

At $\mathrm{BMC}$, research is always in progress.

Learn more biomedcentral.com/submissions 